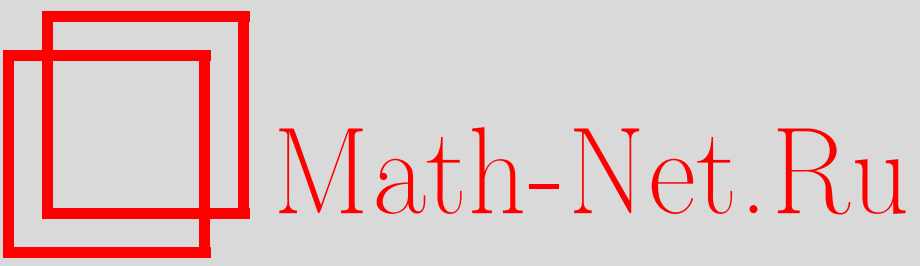

И. А. Пушкарь, О предельных циклах, рождающихся при возмущении гамильтоновых систем, УМH, 2002, том 57, выпуск 5, 161-162

DOI: https://doi.org/10.4213/rm565

Использование Общероссийского математического портала Math-Net.Ru подразумевает, что вы прочитали и согласны с пользовательским соглашением

http://www . mathnet.ru/rus/agreement

Параметры загрузки:

IP : 54.162 .85 .209

26 апреля 2023 г., 13:00:31 


\title{
О ПРЕДЕЛЬНЫХ ЦИКЛАХ, РОЖДАЮЩИХСЯ ПРИ ВОЗМУЩЕНИИ ГАМИЛЬТОНОВЫХ СИСТЕМ
}

\author{
И. А. ПУШКАРЬ
}

Сколько замкнутых траекторий общего положения гамильтоновой системы на плоскости, гамильтониан которой - полином степени $n$, можно превратить в предельные циклы при помощи малого возмущения системы полиномиальным векторным полем степени $m$ (cp. [1]-[4])?

Результаты настоящей заметки возникли при попытке ответить на этот вопрос. В пп. 2 и 3 формулируются теоремы об абелевых интегралах в комплексной и вещественной областях, справедливые в любой размерности $k$. В п. 4 эти теоремы в случае $k=2$ применяются к поставленному вьше вопросу.

Я благодарна Ю.С. Илњяшенко и А. Г. Хованскому за полезные обсуждения.

1. Полиномы с невырожденной старшей однородной частью. Скажем, что полином $H$ степени $n$ в $\mathbb{C}^{k}$ имеет невырожденную старшую однородную часть $h$, если гиперповерхность, заданная однородным уравнением $h=0$, имеет единственную особую точку - начало координат. Множетсво $A_{H} \subset \mathbb{C}$ критических значений полинома $H$ конечно. Пусть $U=\mathbb{C}^{k} \backslash H^{-1}\left(A_{H}\right)$ - объединение всех неособых поверхностей уровня полинома $H$. Известно, что ограничение полинома $Н$ с невьрожденной старшей однородной частью на мно-

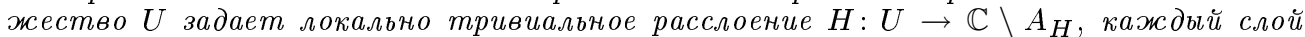
которого имеет гомологический тип букета $(n-1)^{k}$ сфер размерности $(k-1)(\mathrm{cp} .[5])$.

$\mathrm{C}$ некритическим значением $a$ полинома $H$ свяжем универсальную накрьвающую $\pi: Y_{H, a} \rightarrow$ $\mathbb{C} \backslash A_{H}$. Точку $c$ универсальной накрьвающей $Y_{H, a}$ можно отождествлять с классом гомотопически эквивалентных кривых в $\mathbb{C} \backslash A_{H}$ с закрепленными концами $a$ и $\pi(c)$. Поэтому с точкой $c \in Y_{H, a}$ связан изоморфизм $\tau_{c}: H^{k-1}\left(F_{\pi(c)}\right) \rightarrow H^{k-1}\left(F_{a}\right)$ пространств $(k-1)$-мерных когомологий слоев расслоения $H: U \rightarrow \mathbb{C} \backslash A_{H}$. Мы построим две функции, определенные на $Y_{H, a}$ и принимающие значения в пространстве $(k-1)$-мерных когомологий слоя $F_{a}$.

Функция $\Phi_{\omega_{k}}$ строится по любой голоморфной $k$-форме $\omega_{k}$ в $\mathbb{C}^{k}$. Именно, $\Phi_{\omega_{k}}(c)$ определяется как образ при изоморфизме $\tau_{c}$ класса $(k-1)$-мерных когомологий слоя $F_{\pi(c)}$, определенного на поверхности $H=\pi(c)$ формой $\omega_{k} / d H$.

Функция $\Psi_{\alpha_{k-1}}$ строится по любой голоморфной $(k-1)$-форме $\alpha_{k-1}$ в $\mathbb{C}^{k}$. Именно, $\Psi_{\alpha_{k-1}}(c)$ определяется как образ при изоморфизме $\tau_{c}$ класса $(k-1)$-мерных когомологий слоя $F_{\pi(c)}$, определенного на поверхности $H=\pi(c)$ формой $\alpha_{k-1}$.

2. Комплексные теоремы. Для $j=0,1, \ldots$ рассмотрим в $k$-мерном пространстве $\mathbb{R}^{k}$ куб $K_{j}(n, k)$, определенный неравенствами $n j \leqslant x_{1} \leqslant n j+n-2 ; 0 \leqslant x_{i} \leqslant n-2, i=2, \ldots, k$. Обозначим через $K(n, m)$ объединение всех кубов $K_{j}(n, k)$. По определению число $M(n, m, k)$ равно количеству чельх точек множества $K(n, k)$, удовлетворяющих неравенству $\sum_{i=1}^{k} x_{i} \leqslant m$.

Tеорема 1. Пусть $\Omega_{k, m}$ - пространство дифференциальньх $k$-форм $\omega_{k}$ в $\mathbb{C}^{k}$ вида $\omega_{k}=P d x_{1} \wedge \cdots \wedge d x_{k}$, где $P$ - полином степени меньше или равной $m$. Тогда для полинома $H$ степени $n$ с невьрожденной старшей однородной частью размерность линейного пространства функций $\Phi_{\omega_{k}}$, где $\omega_{k} \in \Omega_{k, m}$, равняется $M(n, m, k)$.

Теорема 2. Пусть $\Omega_{k-1, m+1}-$ пространство дифференциальных $(k-1)$-форм $\alpha_{k-1}$ в $\mathbb{C}^{k}$ вида $\alpha_{k-1}=P_{1} d x_{2} \wedge \cdots \wedge d x_{k}+\cdots+P_{k} d x_{1} \wedge \cdots \wedge d x_{k-1}$, где $P_{1}, \ldots, P_{k}-$ полиномь степени меньше или равной $m+1$. Тогда для полинома $H$ степени $n$ с невьрожденной старшей однородной частью размерность линейного пространства функций $\Psi_{\alpha_{k-1}}$, где $\alpha_{k-1} \in \Omega_{k-1, m+1}$, равняется $M(n, m, k)$.

С каждым циклом $\gamma \in H_{k-1}\left(F_{a}\right)$ и голоморфной $(k-1)$-формой $\alpha_{k-1}$ в $\mathbb{C}^{k}$ свяжем комплекснозначную функцию $\Phi_{\alpha_{k-1}}(\gamma)$ на универсальной накрывающей $Y_{H, a}$, сопоставляющую точке $c \in Y_{H, a}$ число, равное значению класса когомологий $\Psi_{\alpha_{k-1}}(c)$ на цикле $\gamma$.

ТЕорема 3. Пусть $H$-ультраморсовский полином (т.е. $Н$ имеет невырожденную 
старшую однородную часть и морсовские критические точки с различными критическими значениями) степени $n$. Пусть цикл $\gamma \in H_{k-1}\left(F_{a}\right)$ не лежит в ядре формь пересечения слоя $F_{a}$. Тогда размерность пространства функиий $\Psi_{\alpha_{k-1}}(\gamma)$, где $\alpha_{k-1} \in \Omega_{k-1, m+1}$, равняется $M(n, m, k)$.

3. Вещественные теоремы. Неособая компонента связности поверхности уровня $H=c$ в $\mathbb{R}^{k}$ вещественного полинома $H$ ориентирована как граница области $H \leqslant c$. С вещественным полиномом $H$ в $\mathbb{R}^{k}$ свяжем множество $\Gamma_{H}$ всех компактных неособых компонент связности всевозможных поверхностей уровня этого полинома, наделенных описанной выше ориентацией. Множество $\Gamma_{H}$ имеет естественную структуру вещественно аналитической кривой.

$\mathrm{C}$ диффференциальной $(k-1)$-формой $\alpha_{k-1}$ на $\mathbb{R}^{k}$ свяжем функцию $I_{\alpha_{k-1}}$ на $\Gamma_{H}$. Значение $I_{\alpha_{k-1}}$ в точке а - это $\int_{\gamma_{a}} \alpha_{k-1}$, где $\gamma_{a}-$ чикл в $\mathbb{R}^{k}$, соответствующий $а$.

Обозначим через $\mathbb{R} \Omega_{k-1, m+1}$ пространство вещественных дифференциальных $(k-1)$-форм в $\mathbb{R}^{k}$ вида $\alpha_{k-1}=P_{1} d x_{2} \wedge \cdots \wedge d x_{k}+\cdots+P_{k} d x_{1} \wedge \cdots \wedge d x_{k-1}$, где $P_{1}, \ldots, P_{k}$ - вещественные полиномы степени меньше или равной $m+1$.

Теорема 4. Пусть полином $H$ имеет степень меньше или равную $n$. Тогда размерность пространства функиий $I_{\alpha_{k-1}}$, соответствующих формам $\alpha_{k-1} \in \mathbb{R} \Omega_{k-1, m+1}$, меньше или равна $M(n, m, k)$.

ТЕОРема 5. Пусть полином $H$ степени $n>k$ является ультраморсовским и множество $\Gamma_{H}$ непусто. Тогда размерность пространства функций $I_{\alpha_{k-1}}$, соответствующих формам $\alpha_{k-1} \in \mathbb{R} \Omega_{k-1, m+1}$, равна $M(n, m, k)$. При этом если функция $I_{\alpha_{k-1}}$ тождественно равна нулю на одной из компонент связности кривой $\Gamma_{H}$, то она тождественно равна нулю на всей кривой.

4. Приложения. Пусть $H$ - полином от двух переменных. Набор из $p$ точек $\Gamma_{H}$ назовем $m$-хорошим, если существует $\alpha_{1} \in \mathbb{R} \Omega_{1, m}$, для которой $I_{\alpha_{1}}$ имеет в точках набора некратные нули. Набор из компактных неособых компонент связности линий уровня $H$ на плоскости назовем $m$-хорошим, если соответсвующий ему набор точек на $\Gamma_{H}$ является $m$-хорошим.

Рассмотрим негамильтоново возмущение $v=v_{H}+\delta j\left(\alpha_{1}\right)$ гамильтонова векторного поля $v_{H}$ с гамильтонианом $H$ на плоскости со стандартной симплектической структурой, где $j$ - изоморфизм кокасательного и касательного пространств, соответствующий симплектической структуpe, $\delta$ - вешественное число и $\alpha_{1}$ - 1-форма, фигурирующая в определении $m$-хорошего набора $\gamma_{1}, \ldots, \gamma_{p}$. Согласно критерию Пуанкаре-Понтрягина (см. [1]) при малом $\delta$ негамильтоново векторное поле $v$ имеет предельные циклы, расположеные вблизи кривых $\gamma_{1}, \ldots, \gamma_{p}$. Поэтому вопрос, сфформулированньй в начале заметки, приводит к Основной ЗАДАчЕ: для полинома $H$ от двух переменных и для натурального числа $m$ найти наибольшее натуральное число $q(H, m)$ такое, что в пространстве наборов из $p=q(H, m)$ точек кривой $\Gamma_{H}$ всюду плотны т-хорошие наборы.

Теорема 6. Для любого полинома $H$ от двух переменных степени не выше $n$ в пространстве наборов из $M(n, m-1,2)$ точек кривой $\Gamma_{H} m$-хорошие наборы нигде не плотны.

Tеорема 7. В пространстве полиномов $H$ степени $n$, для которого $\Gamma_{H} \neq \varnothing$, открытое всюду плотное множетсво составляют ультраморсовские полиномы. Для каждого такого ультраморсовского полинома $H$ справедливо равенство $q(H, m)=$ $M(n, m-1,2)-1$.

\section{СПИСОК ЛИТЕРАТУРЫ}

[1] Л. С. Понтрягин // ЖЭТФ. 1934. Т. 4. № 8. С. 234-238. [2] Ю. С. Ильяшенко // Матем. сб. 1969. Т. 80. № 3. С. 388-404. [3] И. А. Пушкарь // Функц. анализ и его прил. 1997. Т. 31. № 2. C. 34-44. [4] L. Gavrilov // Bull. Sci. Math. 1998. V. 122. № 8. P. 571-584. [5] В. И. Apнольд, А. Н. Варченко, С. М. Гусейн-Заде. Особенности дифференцируемых отображений. 2. М.: Наука, 1984. 